\title{
Juvenile Xanthogranuloma on the Auricle: Unusual Case Report and Clinico-Dermoscopic Correlation
}

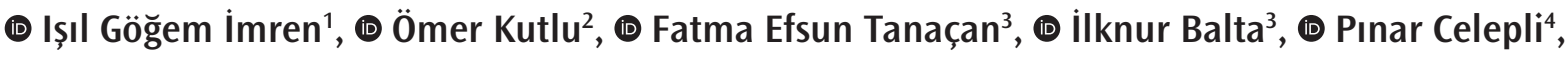 \\ (D) Hatice Meral Ekșioğlu³
}

1Pamukkale University Faculty of Medicine, Department of Dermatology and Venereology, Denizli, Turkey

2Usak University Faculty of Medicine, Department of Dermatology and Venereology, Usak, Turkey

${ }^{3}$ Ankara Training and Research Hospital, Clinic of Dermatology and Venereology, Ankara, Turkey

${ }^{4}$ Ankara Training and Research Hospital, Clinic of Pathology, Ankara, Turkey

\section{ABSTRACT}

Juvenile xanthogranuloma (JXG) is a rare and benign proliferative disease, which is the most common form of the non-Langerhans group of histiocytic disorders. We describe a case of JXG with a rather unusual clinical presentation and highlight the importance of using dermoscopy (a "setting sun" pattern) as a non-invasive adjuvant tool in the diagnosis of JXG in a female patient presenting with an eight-month history of multiple auricular nodules. Due to it being a self-regressing disorder, accurate diagnosis of JXG by dermoscopy is crucial and confers advantages by avoiding unnecessary invasive procedures and aggressive treatment for children.

Keywords: Juvenile xanthogranuloma, Dermoscopy, "Setting sun” Pattern

\section{Introduction}

Juvenile xanthogranuloma (JXG) is a rare and benign proliferative disease, which is the most common form of the non-Langerhans group of histiocytic disorders [1,2]. It was first described by Adamson [3] in 1905, who presented a child that developed numerous yellow-white papules on the body in the first two weeks of life. However, widespread recognition of an entity resembling JXG occurred in 1954 with Helwig and Hackney [4]. JXG appears predominantly in the childhood period, especially in the first two years of life, despite there being adult cases. It is usually characterized by one or multiple yellow-pink cutaneous nodules and less often additional lesions can involve extracutaneous tissues.

The etiopathogenesis of xanthogranuloma is not exactly known, but the disease is believed to be a reactive granulomatous process against undetermined physical or infectious stimuli rather than neoplasia. The prognosis of patients with cutaneous involvement is excellent, with spontaneous regression, but in some cases atrophic residual hyperpigmented scars may remain. The diagnosis of JXG is clinically based and supported by histopathology. The dermoscopic examination also provides clues and allows distinguishing from other possible diseases.

We describe a case of JXG with a rather unusual clinical presentation and highlight the importance of using dermoscopy (a "setting sun" pattern) as a non-invasive adjuvant tool in the diagnosis of JXG in a female patient presenting with an eight-month history of multiple auricular nodules. Due to it being a self-regressing disorder, accurate diagnosis of JXG by dermoscopy is crucial and confers advantages by avoiding unnecessary invasive procedures and aggressive treatment for children.

\section{Case Report}

We report the case of a seven-year-old female patient, presenting with non-tender, mobile yellowish-red multiple nodules over the 
helical rim of her left ear for eight months (Figure 1). Her personal and family histories revealed no relevant findings. In addition, she had no history of any trauma or infection. On dermatological examination, the left ear was remarkable for three well-defined yellow-red nodules, including the largest one $10 \mathrm{~mm} \times 5 \mathrm{~mm}$ over the helical rim. The nodules were shiny, with some evidence of telangiectasia and scaling located centrally. There was no ulceration of the overlying skin, it did not appear to be fixed and no enlarged lymph nodes were palpated.

On dermoscopy, we observed a red-yellow structureless center with a pale erythematous halo, characterizing the "setting sun" pattern, as well as linear and branched telangiectasias, which are characteristic features of JXG (Figure 2). Although these were typical dermoscopic findings for JXG, due to clinical features of the lesions, the case was compatible with angiolymphoid hyperplasia with eosinophilia. Therefore, we performed a punch biopsy. Histopathologic examination revealed mild hyperkeratosis and acanthosis in the epidermis and histiocytic infiltrate in the dermis, as well as an eosinophilic infiltrate with a few lymphocytes mixed with multinucleated Touton giant cells. Touton giant cells were scattered throughout the lesion in association with moderate

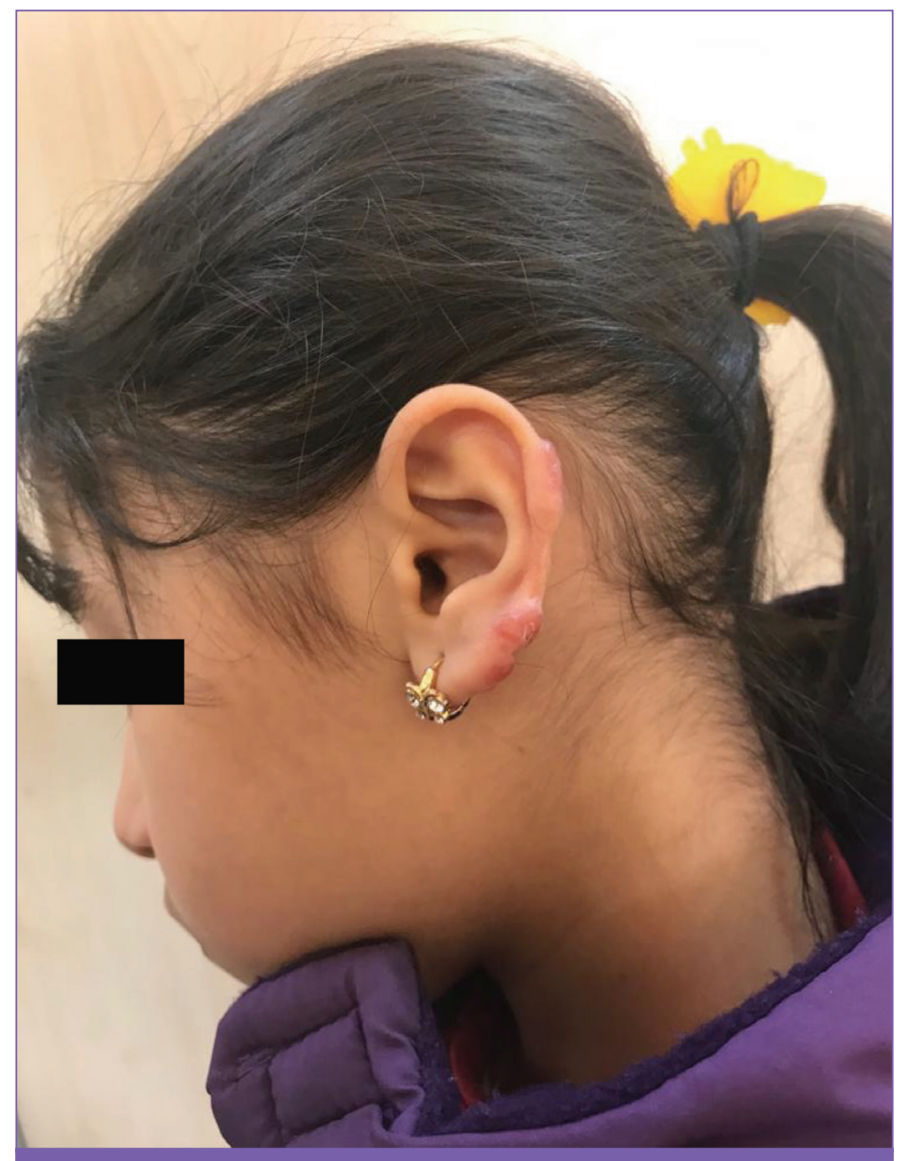

Figure 1. Non-tender, mobile yellowish-red multiple nodules over the helical rim numbers of lymphocytes and histiocytes with a foamy cytoplasm (Figures 3, 4). There was no malignant appearance, such as nuclear atypia, an increased number of nucleoli or necrosis.

In the immunohistochemical analysis of the lesional cells, they were positive for CD68, but negative for S100, CD1a, and langerin. Based on clinical examination, dermoscopic and histological findings, a diagnosis of JXG was made. Complete blood count and biochemical parameters were investigated for possible systemic involvement. An ophthalmic exam was also conducted for ocular JXG since this is the most common type of extracutaneous JXG. Finally, all blood tests were in the normal range and there was no ocular involvement. The patient was followed up due to the self-healing nature of JXG.

\section{Discussion}

The term "histiocyte" refers to large white blood cells resident in tissues, including Langerhans cells, monocytes-macrophages and dendritic cells. This group of diseases has generally been divided into Langerhans cell histiocytosis (LCH) and non-Langerhans

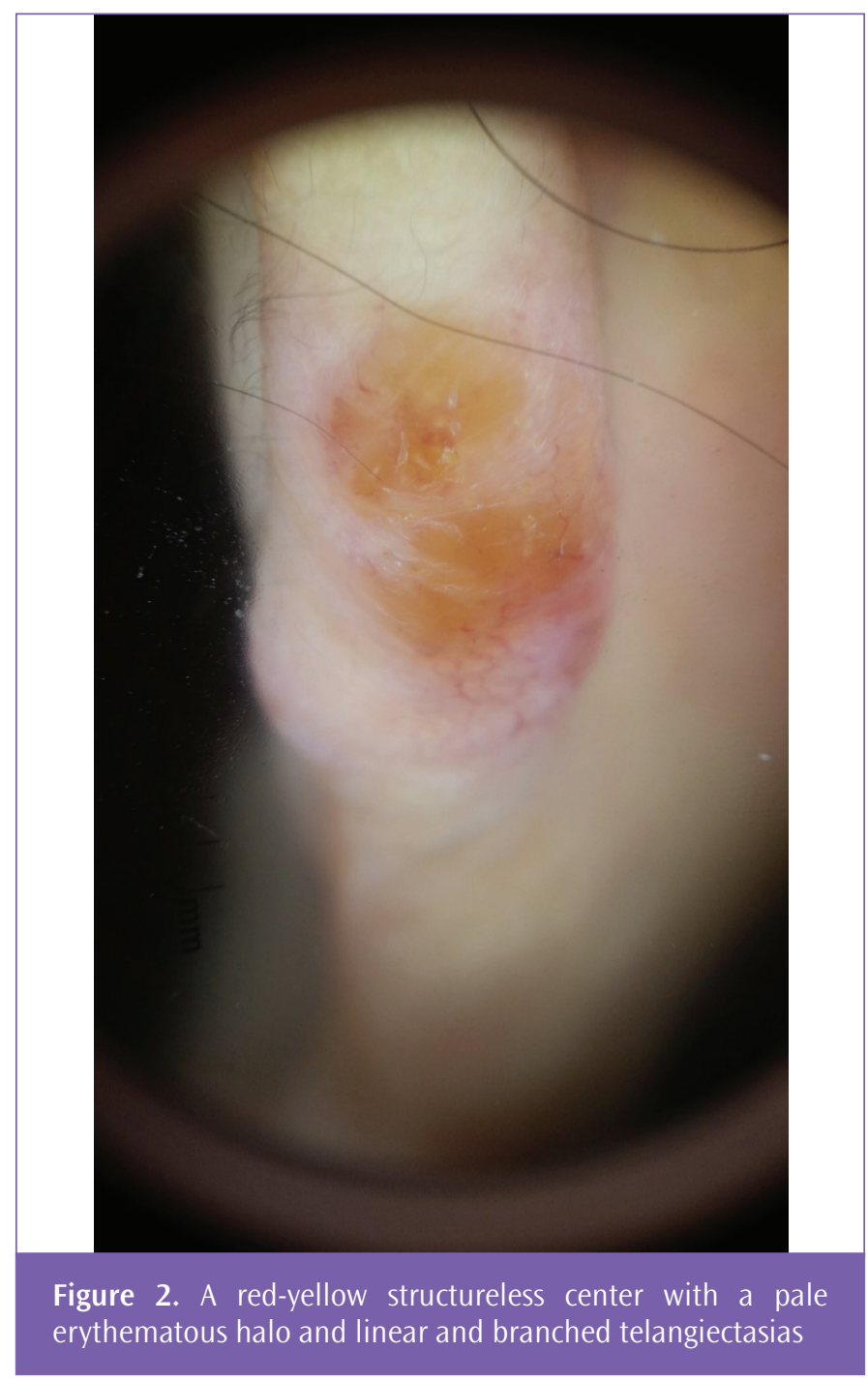


histiocytosis (non-LCH). Langerhans cells are specialized dendritic cells found in the skin and mucosa. In contrast, non-LCH is thought to be derived from the monocyte-macrophage lineage. In LCH there is a predominance of CD1a, langerin, and S100 positive cells. Positivity of histiocytic markers such as CD68, and negativity for CD1a, langerin, and S-100 protein indicate the origin of nonLangerhans cells. JXG is the most common form of non-LCH.

The pathogenesis of JXG remains unclear. It is most likely a reactive granulomatous reaction of histiocytes to unidentified stimuli rather than a neoplastic process. It has been reported that JXG as a disease in which the macrophage response may occur from a non-specific injury $[5,6,7,8]$. It has been reported that JXG represents $0.5 \%$ of all pediatric tumors [2]. The disease usually appears in the first two

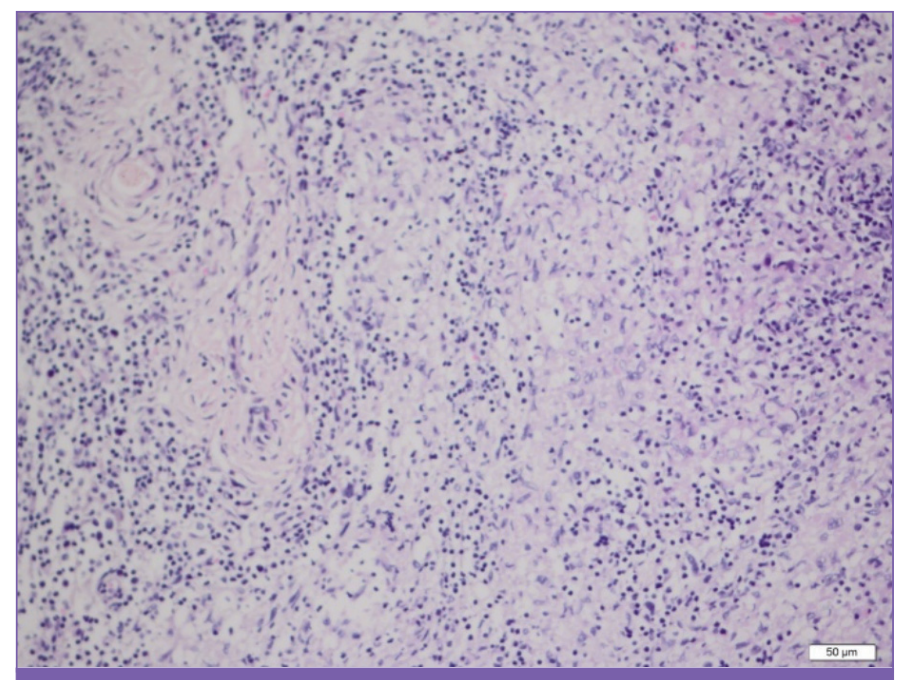

Figure 3. Epitheloid and foamy histiocytes in groups of polymorphic mature lymphocytes in the dermis (hematoxylin and eosin, $\mathrm{x} 100$ )

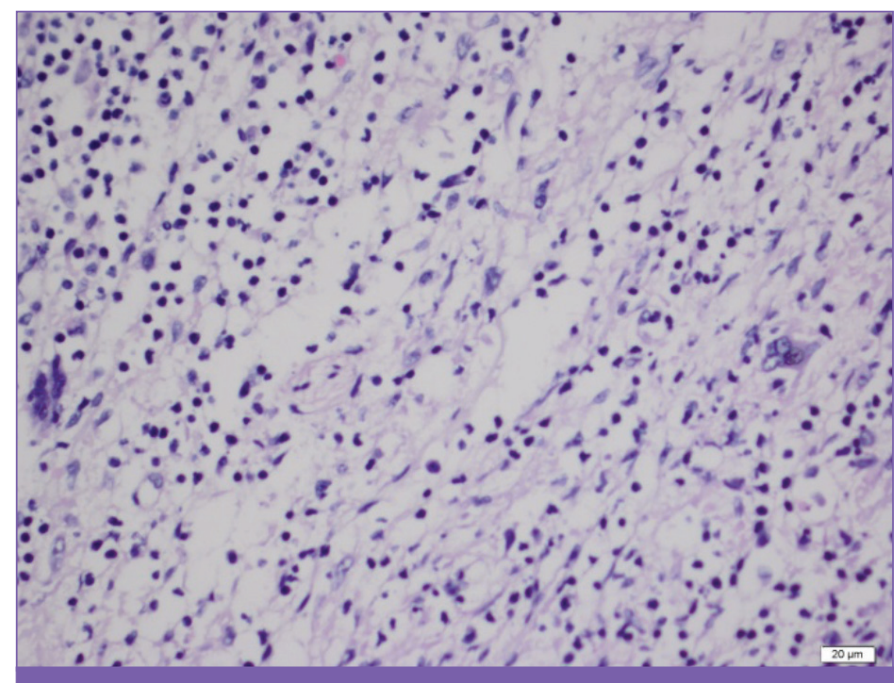

Figure 4. Touton type and foreign body type multinuclear giant cells (hematoxylin and eosin, $x 200$ ) years of life: 5 to $17 \%$ are present at birth and 40 to $70 \%$ appear during the first year $[5,6]$. Despite the term "juvenile", $10 \%$ of cases manifest in adulthood [6].

JXG is characterized by one or multiple 2 to $7 \mathrm{~mm}$ firm, elastic, rounded papules or nodules that initially start as pink-brown and evolve to a xanthomatous yellowish appearance with erythematous halos. Superficial telangiectasias or brownish color may be visible in later stages $[1,6,9]$. Although JXG mostly occurs as a solitary lesion, multiple JXG may occur in 7 to $10 \%$ of the cases, specifically in infants and males [2,6,7]. Skin is the most commonly involved organ for JXG and less often additional lesions may be seen in the deeper soft tissue and visceral organs. Cutaneous JXG mostly occurs in the head and neck, followed by the trunk, limbs and palmoplantar region $[6,7,8]$. Clinical differential diagnosis of JXG includes $\mathrm{LCH}$, mastocytoma, angiolymphoid hyperplasia with eosinophilia, Spitz nevus, and other xanthomatous conditions (papular and tuberous xanthoma, xanthoma disseminatum and eruptive xanthomas).

Dermoscopy has an important role in the non-invasive diagnosis of JXG. Under polarized dermoscopy, the four most commonly dermoscopic features of JXG include a setting sun pattern; clouds of pale yellow globules; a whitish streak; and branched and/or linear vessels $[9,10]$. Moreover, clouds of pale yellowish globules represent lipid-laden xanthomatous histiocytic infiltration in the upper dermis, while a discrete pigment network and whitish streaks indicate areas of fibrosis. The pattern described as setting sun is characterized by a yellow-orange central area, which may show areas of white-yellow clouds and an erythematous halo. JXG has three stages: (i) early evolutionary stage, (ii) fully developed stage, and (iii) late regressive stage. The main observed dermoscopic feature of JXG is the setting sun appearance and surrounding linear or branched vessels, which can be appreciated in the three stages with the whitish streak becoming more prominent and surrounding erythema decreases in the late regressive stage. Clouds of pale yellow globules become more evident in the advanced stages due to the transformation of vacuolated cells to xanthomatous cells, becoming more yellowish across the three stages [11].

\section{Conclusion}

We describe a case of JXG with a rather unusual clinical presentation and highlight the importance of considering this entity in the differential diagnosis of benign soft tissue tumors of the ear. There are only occasional case reports of xanthogranuloma involving the auricle. Due to the self-regressing and benign nature of the disease, and in order to avoid unnecessary surgical procedures, dermoscopy is a useful noninvasive diagnostic technique for clinicians. In addition, follow-up with dermoscopy may prevent invasive procedures due to self-regression and the benign nature of the disease. 


\section{Ethics}

Informed Consent: Consent form was filled out by all participants.

Peer-review: Internally peer-reviewed.

\section{Authorship Contributions}

Concept: I.G.I., Ö.K., Design: I.G.I., Ö.K., Data Collection or Processing: P.C., Analysis or Interpretation I.G.I., Ö.K., F.E.T., I.B., P.C., H.M.E., Literature Search: I.G.I., F.E.T., I.B., Writing: I.G.I.

Conflict of Interest: No conflict of interest was declared by the authors.

Financial Disclosure: The authors declared that this study received no financial support.

\section{References}

1. Weitzman S, Jaffe R. Uncommon histiocytic disorders: the non-Langerhans cell histiocytoses. Pediatr Blood Cancer 2005;45:256-264.

2. Pajaziti L, Hapçiu SR, Pajaziti A. Juvenile xanthogranuloma: a case report and review of the literature. BMC Res Notes 2014;7:174.

3. Adamson HG. Society intelligence: the dermatological society of London. Br J Dermatol 1905;17:222.
4. Helwig EB, Hackney VC. Juvenile xanthogranuloma (nevoxanthoendothelioma). Am J Pathol 1954;30:625-626.

5. Jung T, Emmert S, Günzl HJ, Neumann C, Rünger TM. Congenital manifestations of juvenile xanthogranuloma (large nodular form) Hautarzt 2000;51:423-426.

6. Dehner LP. Juvenile xanthogranulomas in the first two decades of life a clinicopathologic study of 174 cases with cutaneous and extracutaneous manifestations. Am J Surg Pathol 2003:27:579-593.

7. Clayton TH, Mitra A, Holder J, Clark SM. Congenital plaque on the chest. Diagnosis: solitary giant congenital juvenile xanthogranuloma. Clin Exp Dermatol 2007;32:613-614.

8. Chang MW. Update on juvenile xanthogranuloma: unusual cutaneous and systemic variants. Semin Cutan Med Surg 1999;18:195-205.

9. Oliveira TE, Tarlé RG, Mesquita LAF. Dermoscopy in the diagnosis of juvenile xanthogranuloma. An Bras Dermatol 2018;93:138-140.

10. Mun JH, Ohn J, Kim KH. Dermoscopy of giant juvenile xanthogranuloma. J Am Acad Dermatol 2017;76:76-78.

11. Song M, Kim, SH, Jung, DS, Ko HC, Kwon KS, Kim MB. Structural correlations between dermoscopic and histopathological features of juvenile xanthogranuloma. J Eur Acad Dermatol Venereol 2011;25:259-263. 